\title{
Static temperature analysis and compensation of MEMS gyroscopes
}

\author{
Q.J. Tang ${ }^{1,2}$, X.J. Wang ${ }^{1}$, Q.P. Yang ${ }^{2 \star}$, and C.Z. Liu ${ }^{1}$ \\ 1 MOEMS Education Ministry Key Laboratory, Tianjin University, Tianjin 300072, P.R. China \\ 2 School of Engineering and Design, Brunel University, Uxbridge, Middlesex UB8 3PH, UK
}

Received: 29 June 2013 / Accepted: 7 November 2013

\begin{abstract}
MEMS gyroscopes as a kind of angular rate sensor have been widely used, but their accuracy tends to be low in practical applications, especially under temperature influence, and they generally require error compensation. Based on the analysis of gyroscope operating principle, this paper has shown that the resonant frequency and measuring precision of the gyroscope are dependent on temperature and temperature gradients. The paper has thus proposed a compensation model based on temperature and temperature gradients. The experimental results have demonstrated that the thermal drift of zero bias can be effectively suppressed, and the accuracy can be improved by one order of magnitude after compensation. Compared with compensation methods only based on temperature, the new method gives significantly better performance. The new error compensation model has not only integrated the differences under different temperature conditions, but also reduced the repeatability errors. It provides a theoretical basis for accurate compensation of the gyroscope thermal error in practical applications and is applicable to other MEMS gyroscopes.
\end{abstract}

Keywords: MEMS gyroscope; temperature gradients; zero bias compensation; temperature characteristics

\section{Introduction}

In the control systems of airborne photoelectric reconnaissance devices, in order to realize the targets' capture, tracking and measurement, it usually requires keeping the optical axis of photoelectric sensors facing towards the targets. The stabilized platform should help solve two problems: servo tracking and the carrier's vibration isolation. Gyroscopes, as the angular velocity measuring device in space, are the core components of the system. And MEMS (Micro Electromechanical System) gyroscopes have been widely used due to its small size, light weight, low power consumption and low price. Figure 1 shows a commonly used servo control system, which contains two control loops, the position loop and the velocity loop, while some systems perhaps also include the current loop [1].

As the speed sensitive device in the velocity loop, MEMS gyroscopes also sense the carrier's vibration in the inertial space, and output a voltage which is proportional to the angular velocity, then the controller will produce a trimming moment which is equal to the disturbance torque and in the opposite direction to keep the line of sight (LOS) stable. The feedback control of the velocity loop can also increase the bandwidth and the speed of response of the position loop, thus improving the tracking

${ }^{\star}$ Correspondence: Ping.Yang@brunel.ac.uk

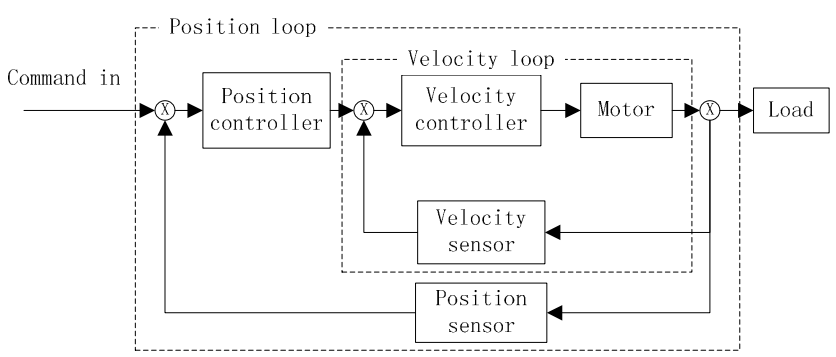

Fig. 1. Servo control system.

performance. However, in practical applications, the error caused by inertial device accounts for the main part of the system error. The noise and drift of gyroscopes reduce the signal resolution, especially when the gyroscope signal is very small. And this results in migration of the LOS, losing the target and resulting in fuzzy images [2], and generally leads to higher requirement for the inertial components. As MEMS gyroscope accuracy is currently low, it is important to perform the testing and calibration of the gyroscope including inertial components, accurate acquisition of relevant data and error compensation to improve the inertial device's accuracy [3]. Since temperature is one of the main error sources of MEMS gyroscopes [4,5], this study aims to establish a new compensation model to enhance the zero-bias accuracy of MEMS gyroscopes. 


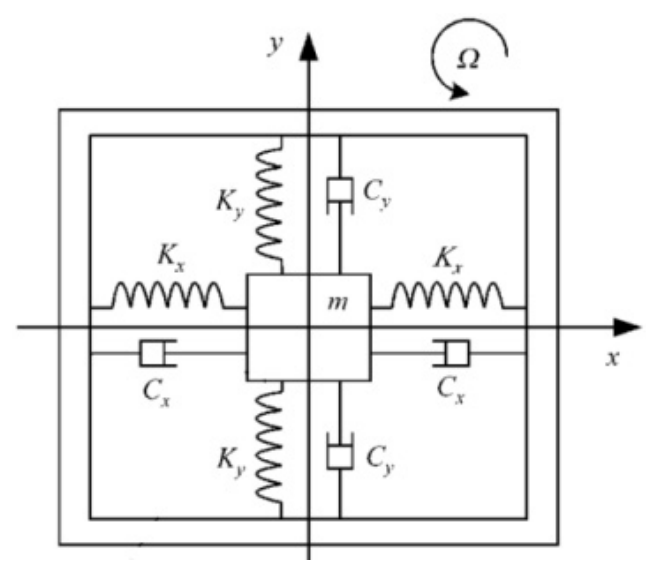

Fig. 2. Schematics of the vibrating mass gyroscope.

\section{Structure and operating mechanism}

The structure of a $z$-axis MEMS gyroscope is shown in Figure 2. It has two perpendicular vibration directions, $x$-axis and $y$-axis, which correspond to drive mode and test mode respectively. The $y$-axis vibration (test mode) is caused by Coriolis force. When the gyroscope is powered on, the mass block will vibrate harmonically in $x$-axis direction due to periodic electrostatic force. Given an angular velocity along the $z$-axis, $\Omega$, the mass will sense the Coriolis force in the $y$-axis. And the angular velocity, $\Omega$, can be calculated through the mass displacement in the $y$-direction [6-8].

According to Newton's second law of motion, it can be obtained in $x$-axis and $y$-axis:

$$
\left\{\begin{aligned}
m_{x} \ddot{x}+c_{x} \dot{x}+ & {\left[k_{x}-m_{x}\left(\Omega_{y}^{2}+\Omega_{z}^{2}\right)\right] x } \\
& -2 m_{x} \Omega_{z} \dot{y}+m_{x} \Omega_{x} \Omega_{y} y=f_{d}+M_{x} \\
m_{y} \ddot{y}+c_{y} \dot{y}+ & {\left[k_{y}-m_{y}\left(\Omega_{x}^{2}+\Omega_{z}^{2}\right)\right] y } \\
+ & 2 m_{y} \Omega_{z} \dot{x}+m_{y} \Omega_{x} \Omega_{y} x=M_{y}
\end{aligned}\right.
$$

where $m_{i}$ is the mass, $c_{i}$ the damping coefficient, $k_{i}$ the stiffness of the elastic beams and $M_{i}$ the interference force, all in $i$-axis direction $(i=x$ or $y) ; f_{d}$ is the electrostatic driving force. If $f_{d}$ is ideal and harmonic, its driving equation can be represented as: $f_{d}=f_{e} \sin \omega_{t}$.

When the $z$-axis gyroscope rotates about the $z$-axis without any interference force, i.e. $\Omega_{x}=\Omega_{y}=0, M_{x}=$ $M_{y}=0$, equation (1) can be written as:

$$
\left\{\begin{array}{l}
m_{x} \ddot{x}+c_{x} \dot{x}+\left(k_{x}-m_{x} \Omega_{z}^{2}\right) x-2 m_{x} \Omega_{z} \dot{y}=f_{d} \\
m_{y} \ddot{y}+c_{y} \dot{y}+\left(k_{y}-m_{y} \Omega_{z}^{2}\right) y+2 m_{y} \Omega_{z} \dot{x}=0
\end{array}\right.
$$

or

$$
\left\{\begin{array}{l}
\ddot{x}+2 \xi_{x} \omega_{x} \dot{x}+\left(\omega_{x}^{2}-\Omega_{z}^{2}\right) x=\frac{f_{d}}{m_{x}}+2 \Omega_{z} \dot{y} \\
\ddot{y}+2 \xi_{y} \omega_{y} \dot{y}+\left(\omega_{y}^{2}-\Omega_{z}^{2}\right) y=-2 \Omega_{z} \dot{x}
\end{array}\right.
$$

where $\omega_{x}=\sqrt{\frac{k_{x}}{m_{x}}}$ is the natural frequency of the drive mode; $\xi_{x}=\frac{c_{x}}{2 \omega_{x} m_{x}}$ is the damping ratio of the drive mode; $\omega_{y}=\sqrt{\frac{k_{y}}{m_{y}}}$ is the natural frequency of the test mode; $\xi_{y}=\frac{c_{y}}{2 \omega_{y} m_{y}}$ is the damping ratio of the test mode.

Since the natural frequencies of drive mode and test mode are much larger than the angular velocity, $\Omega_{z}$, the latter can be ignored on the left side of equation (3). The $y$-axis is governed by a traditional force model, although the Coriolis force induced by the $x$-axis should be considered. In the actual design of closed-loop driving circuit, the Coriolis force which causes vibration in driving direction should be cancelled. Thus the above equation can be further simplified as $[8,9]$ :

$$
\left\{\begin{array}{l}
\ddot{x}+2 \xi_{x} \omega_{x} \dot{x}+\omega_{x}^{2} x=\frac{f_{d}}{m_{x}} \\
\ddot{y}+2 \xi_{y} \omega_{y} \dot{y}+\left(\omega_{y}^{2}-\Omega_{z}^{2}\right) y=-2 \Omega_{z} \dot{x} .
\end{array}\right.
$$

\section{Temperature characteristic analysis}

Silicon as a kind of thermo-sensitive material is greatly influenced by temperature. Its properties including structure, size, elasticity modulus and residual stress, etc. will change with temperature. The change of its elasticity modulus is particularly significant as it will cause the change of system stiffness, resulting in the change of resonance frequency. Temperature change will thus lead to the drift of gyroscope's resonance frequency $[7,8]$.

Elasticity modulus has an approximate linear relationship with temperature change [7-12]:

$$
E(T)=E\left(T_{0}\right)-k_{E} E\left(T_{0}\right)\left(T-T_{0}\right)
$$

where $E(T)$ and $E\left(T_{0}\right)$ are the elasticity modulus of silicon at the temperature of $T$ and $T_{0}=300 \mathrm{~K} ; k_{E}$ is the temperature coefficient of elasticity modulusof silicon.

Since the MEMS gyroscope system stiffness is proportional to the elasticity modulus, thus we have:

$$
K=K_{0}-k_{E} K_{0}\left(T-T_{0}\right)
$$

where $K$ and $K_{0}$ are the system stiffness at the temperature of $T$ and $T_{0}$, with a unit of $\mathrm{N} / \mathrm{mm}$.

If the temperature $T_{1}$ is close to $T_{2}$, the two temperature coefficients are approximately equal and hence:

$$
K_{2}=K_{1}-k_{E} K_{1}\left(T_{2}-T_{1}\right)
$$

where $K_{2}$ and $K_{1}$ are the system stiffness at the temperature of $T_{2}$ and $T_{1}$. The relationship between resonant frequency and temperature can then be expressed as:

$$
\omega_{n}\left(T_{2}\right)=\sqrt{K_{2} / m}=\sqrt{\left[K_{1}-k_{E} K_{1}\left(T_{2}-T_{1}\right)\right] / m}
$$

where $\omega_{n}\left(T_{2}\right)$ is the gyroscope's resonant frequency at the temperature of $T_{2}, m$ is the weight of the mass block. As $T_{1}$ is close to $T_{2}$, equation (8) can be approximated as:

$$
\omega_{n}\left(T_{2}\right)=\omega_{n}\left(T_{1}\right)\left[1-k_{E}\left(T_{2}-T_{1}\right) / 2\right] .
$$

This shows that gyroscope's resonant frequency is dependent on temperature and temperature gradient. Gyroscope compensation mainly deals with scale factor error 


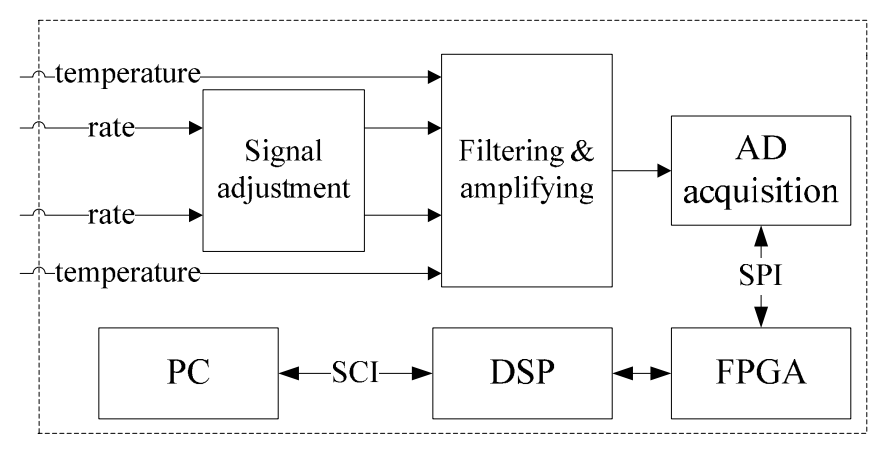

Fig. 3. Schematic diagram of the DAS.

and zero bias error. If the gyroscope is static, the offset of gyroscope detection sensitivity is mainly zero drift. And the chief reason for gyroscope zero bias is the offset of its natural frequency and $Q$-value [8]. When compensating for static error (mainly zero bias error), the commonly used model is based on the built-in temperature sensor, without considering the temperature gradients and the relationship between environment temperature and internal temperature [13].

\section{Temperature compensation model and testing procedure}

\subsection{Temperature compensation model}

In practical applications, MEMS gyroscopes only have internal temperature sensors without any measurement of the environment temperature. In this paper, the temperature gradient is used to represent the difference between internal temperature and environment temperature. The temperature compensation model may be shown as:

$$
F(T)=f(T, \Delta T)+\xi
$$

where $F(T)$ is the zero bias compensation at the temperature of $T ; f(T, \Delta T)$ is the function about temperature $T$ and temperature gradients $\Delta T ; \xi$ is the random error $\xi \sim N\left(0, \sigma^{2}\right)$. To simplify the calculation $f(T, \Delta T)$ is obtained by linear regression analysis. A more specific model used in this paper is:

$$
F(T)=a_{1} T^{2}+a_{2} T+a_{3} \Delta T+a_{4} T \Delta T+a_{5}+\xi
$$

where $a_{1}, a_{2}, a_{3}, a_{4}$ and $a_{5}$ are the coefficient constants.

\subsection{Data acquisition system}

Figure 3 is the schematic diagram of the data acquisition system, which has two channels of gyroscope input interface, with each including rate and temperature signals. The signals enter the AD acquisition module after adjustment, filtering and amplification. The acquired data is then transferred to the DSP through the FPGA. During the testing process, the data is sent to the PC from the DSP through a serial port.

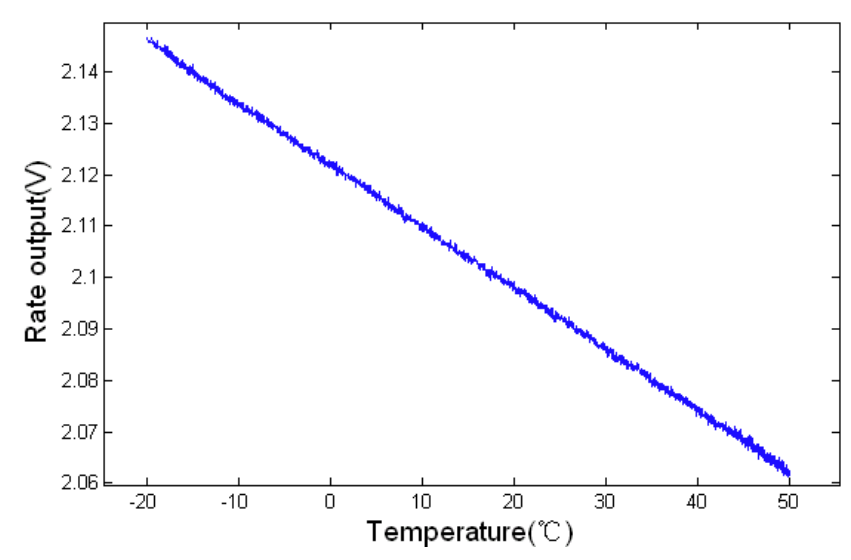

Fig. 4. The relationship between rate output and temperature (7th set of data).

\subsection{Testing procedure}

The data is acquired when the MEMS gyroscope is stable and at different rates of heating up, with the temperature changing from $-20{ }^{\circ} \mathrm{C}$ to $50{ }^{\circ} \mathrm{C}$ over seven heating up times ranging from $5 \mathrm{~min}$ to $60 \mathrm{~min}$. The testing procedure is as follows:

1) Place the MEMS gyroscope in a high-low temperature chamber. Set the temperature at $-20{ }^{\circ} \mathrm{C}$. When the temperature reaches $-20{ }^{\circ} \mathrm{C}$, switch on the gyroscope and data acquisition system. Wait for $1 \mathrm{~h}$.

2) Set the chamber temperature at $50{ }^{\circ} \mathrm{C}$ and heating up time at $5 \mathrm{~min}$. Start to collect gyroscope data. When the chamber temperature reaches $50{ }^{\circ} \mathrm{C}$, wait for $1 \mathrm{~h}$.

3) Collect the gyroscope data within one hour time. Then disconnect the power supply. When the chamber temperature recovers normal temperature, wait for $1 \mathrm{~h}$.

4) Repeat steps $1 \sim 3$ and change the heating up time in step 2 to $10 \mathrm{~min}, 20 \mathrm{~min}, 30 \mathrm{~min}, 40 \mathrm{~min}, 50 \mathrm{~min}$ or $60 \mathrm{~min}$, respectively.

\section{Results and discussion}

According to the above testing procedure, 7 sets of data were obtained. Figure 4 is the 7 th set of data, showing a linear relationship between the zero-bias voltage and the temperature. Thus the data can be analysed by means of linear regression analysis.

All the coefficients in equation (11) can be obtained through the linear regression analysis, as shown in Table 1.

Table 1 illustrates that temperature and temperature gradients of first degree have much higher significant impacts on the zero bias than the quadratic terms. Taking an average of these values under different conditions, we have the final compensation equation:

$$
\begin{aligned}
F(T)= & 0.00000304348 T^{2}-0.25259 T-5.13663 \Delta T \\
& +0.000701 T \Delta T+10381.16
\end{aligned}
$$


Table 1. Values of the five coefficients at different heating up times.

\begin{tabular}{cccccc}
\hline $\begin{array}{c}\text { Heating } \\
\text { up time } \\
\text { (min) }\end{array}$ & $a_{1}$ & $a_{2}$ & $a_{3}$ & $a_{4}$ & $a_{5}$ \\
\hline 5 & 0.0000019504876 & -0.23537397 & -7.4260978 & 0.00098190674 & 10315.948 \\
10 & 0.000002676828 & -0.24618153 & -3.6553626 & 0.00044286063 & 10354.223 \\
20 & 0.0000041203692 & -0.27028934 & -10.03044 & 0.0013282806 & 10453.302 \\
30 & 0.0000038654453 & -0.26552597 & -5.6036385 & 0.00076193292 & 10430.86 \\
40 & 0.0000021652523 & -0.23869247 & 2.9620516 & -0.00037816028 & 10326.501 \\
50 & 0.0000024462827 & -0.24380525 & -3.1428874 & 0.00039041132 & 10347.9 \\
60 & 0.0000040797022 & -0.26828756 & -9.0600264 & 0.001377608 & 10439.366 \\
\hline
\end{tabular}

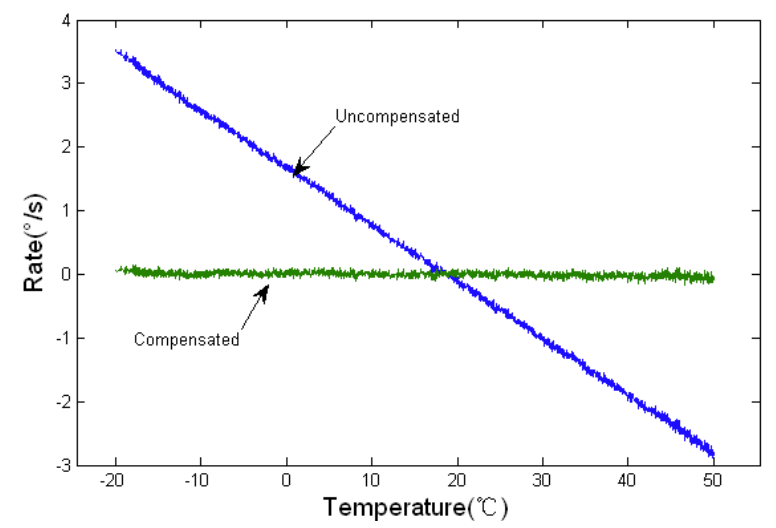

(a)
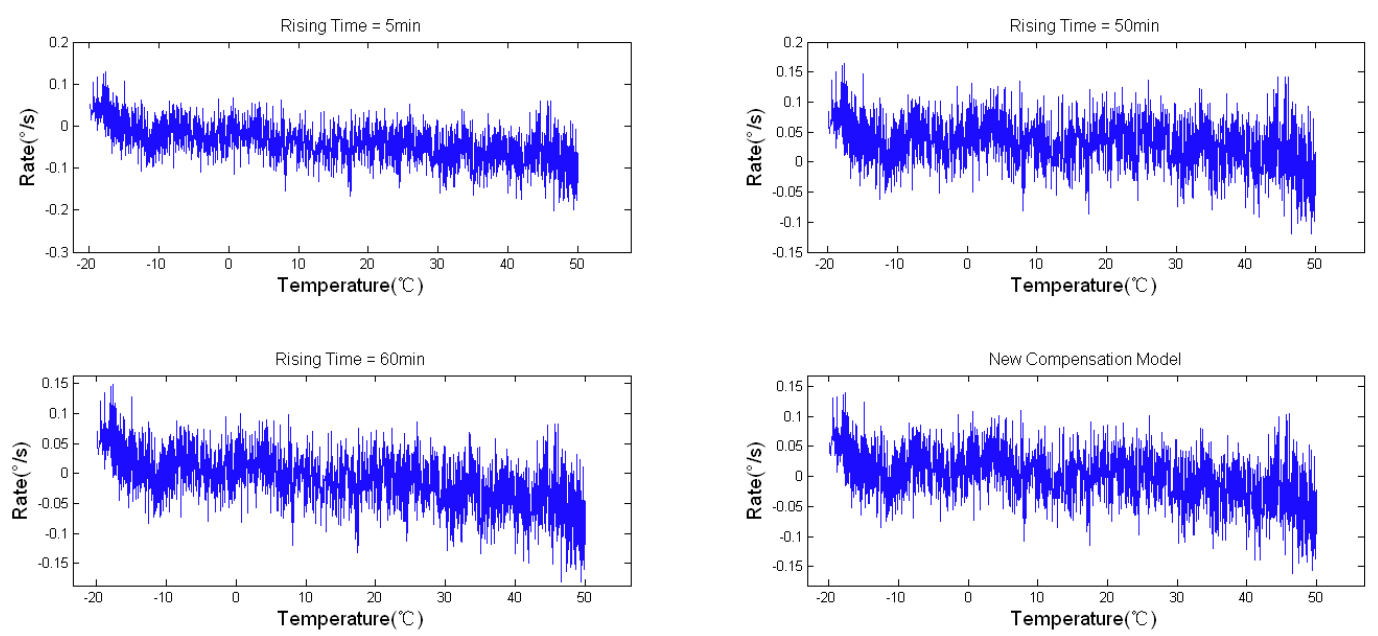

(b)

Fig. 5. Compensation results. (a) Compensation effect with the new fitting model, (b) comparison of compensation results using four different models.

Get three other compensation models by polynomial fitting based on the 1st, 6th and 7th set of data which are based on only temperature, as shown in equation (13).

$$
\left\{\begin{array}{l}
F(T)_{1}=0.0000006733107 T^{2}-0.2146058 T+10232.25 \\
F(T)_{6}=0.0000002464003 T^{2}-0.2437495 T+10346.21 \\
F(T)_{7}=0.0000002043488 T^{2}-0.2357130 T+10311.63
\end{array}\right.
$$

Another set of data were acquired for validation, which has a heating up time of $60 \mathrm{~min}$. The compensation effect of the new model and the compensation results using the four models are compared as shown in Figure 5.

The average and mean square residual errors of the fitting are shown in Table 2.

Figure 5 and Table 2 illustrate that the zero bias of MEMS gyroscope is well compensated by the four compensation models, with the accuracy improved by one order 
Table 2. Average and mean square errors of the four compensation models.

\begin{tabular}{ccccc}
\hline & 1st set & 6 th set & 7th set & New model \\
\hline Average error & -0.0357 & 0.0321 & -0.0079 & 0.0014 \\
Mean square error & 0.0598 & 0.0512 & 0.0482 & 0.0430 \\
\hline
\end{tabular}

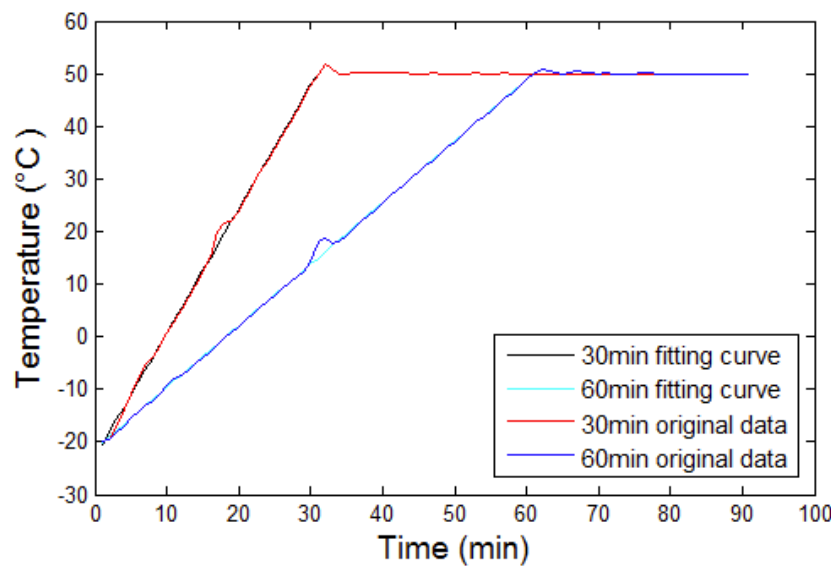

Fig. 6. Chamber temperature variations during $30 \mathrm{~min}$ and 60 min heating processes.

of magnitude before and after compensation. In Table 2, the mean square errors are nearly the same, with the new compensation model giving the minimum value. For average error, the first two compensation models are based on $5 \mathrm{~min}$ and $50 \mathrm{~min}$ heating up time respectively, with similar magnitudes of the average error. The third compensation model is based on 60 min heating up time, which is the same as the validation data, giving a much smaller average error. Using the new compensation model proposed in this paper, the average error is only $0.0014(\% / \mathrm{s})$. Due to different weathers and ambient temperatures, the heating up processes of MEMS gyroscope can vary. In practical calibration process, actual operating environment cannot be repeated. The new compensation model proposed in this paper, which is based on temperature and temperature gradients, integrates different temperature variations and consequently achieves better results.

The measurement system accuracy can be affected in a number of ways. As shown in equation (11), the compensation result is closely related to $T$ and $\Delta T$. In practical use, the MEMS gyroscopes tend to be installed in a confined space and the internal temperature will normally increase until a stable temperature is reached. The heating and cooling processes typically have different responses, resulting in some degree of hysteresis. In the heating process, the control accuracy of the temperature chamber, the linearity of heating up process and the actual temperature field distribution in the chamber will cause some errors to the model presented in this paper. According to the instruction manual, the temperature chamber has a measuring range of $-70{ }^{\circ} \mathrm{C}$ to $150{ }^{\circ} \mathrm{C}$, with a temperature stability of $\pm 0.2^{\circ} \mathrm{C}$ and a temperature uniformity of $\pm 0.5{ }^{\circ} \mathrm{C}\left(-70{ }^{\circ} \mathrm{C} \sim 80{ }^{\circ} \mathrm{C}\right)$. In the practical measurement, however, it is impossible to measure all the points in the chamber, the uniformity will affect the heating linearity, which will cause $\Delta T$ error. Figure 6 shows the temperature variations recorded in the chamber during heating processes of $30 \mathrm{~min}$ and $60 \mathrm{~min}$, respectively. By means of linear regression analysis on the rising segments, the two slopes are found as $2.36{ }^{\circ} \mathrm{C} / \mathrm{min}$ and $1.17^{\circ} \mathrm{C} / \mathrm{min}$, and the standard deviations of the residual errors are $0.84{ }^{\circ} \mathrm{C}$ and $0.57^{\circ} \mathrm{C}$, which are acceptable in this experiment.

Meanwhile, during the operation of the chamber, the small vibration caused by the air flow will also introduce measurement error. In addition, with the increase of the measurement duration, the gyroscope will exhibit errors associated with the gyroscope's fundamental characteristics other than temperature, such as angle random walk, rate random walk, bias instability, Markov noise, etc., which can be further compensated for with stochastic error theories.

\section{Conclusions}

Based on the analysis of the internal structure and operating principle of the MEMS gyroscopes, this paperhas discussed their static temperature characteristics and derived that the gyroscope's zero bias is dependent on temperature and temperature gradient. A new compensation model has thus been established. The testing results indicate that the new model can improve gyroscope's accuracy by one order of magnitude after compensation. Compared with the common compensation methods only based on temperature, the average error can be reduced $7 \sim 20$ times and the standard deviation has declined to less than $80 \%$. The new error compensation model thus not only integrates the different models under different temperature conditions, but also reduces the repeatability error. It provides theoretic basis for the accurate compensation of the gyroscope in practical applications and is applicable to other MEMS gyroscopes.

Acknowledgements. The work was supported by Support Program of National Ministry of Education of China (No. 625010110) and National Natural Science Foundation of China (No. 61179043).

\section{References}

1. X. Pang, D. Fan, X. Teng, J. Peng, Realization of digital airborne opto-electronic servo-system, Opto-Electron. Eng. 34, 10-36 (2007)

2. W. Zhang, D. Fan, Z. Zhang, K. Li, Research on application of MEMS gyro to servo system for E-O stabilization and tracking devices, Opt. Precis. Eng. 14, 689-696 (2006)

3. J. Yang, G. Yuan, L. Xu, The research of testing and calibration technology based on micro-mechanical gyro, Chin. J. Sens. Actuat. 19, 2264-2267 (2006)

4. J. Fang, J. Li, W. Sheng, Improved temperature errormodel of silicon MEMS gyroscopewith inside frame driving, J. Beijing University of Aeronautics and Astronautics 32, 1277-1303 (2006) 
5. U.A. Dauderstadt, P.M. Sarro, P.J. French, Temperature dependence and drift of a thermal accelerometer, International Conference on Solid Sensors and Actuators, Chicago, USA, 1997, pp. 1209-1212

6. V. Annovazzi-Lodi, Mechanical-thermal noise in micromachined gyros, Microelectron. J. 30, 1227-1230 (1999)

7. H. Man, D. Xiao, X. Wu, Z. Chen, Z. Hou, Research on temperature characteristic of mode frequency of silicon micro-gyroscope, Chin. J. Sens. Actuat. 22, 1117-1121 (2009)

8. G. Xia, S. Wang, B. Yang, Research and test on silicon micro-gyroscope temperature characteristics, Meas. Control Technol. 28, 9-13 (2009)

9. S. Gao, H. Liu, Micro electro mechanical system mechanics (National Defence Industrial Press, Beijing, 2008)
10. X. Li, T. Ono, Y. Wang, M. Esashi, Study on ultrathin NEMS cantilevers-high yield fabrication and size effect on young's modulus of silicon, The Fifteenth IEEE International Conference on Micro Electro Mechanical Systems, Las Vegas, USA, 2002, pp. 427-430

11. Y. Tan, H. Yu, Q. Huang, T. Liu, Effect of temperature on the young's modulus of silicon nano-films, Chin. J. Electron Devices 30, 755-758 (2007)

12. H. Chen, R. Zhang, B. Zhou, Z. Chen, Research on thermal characteristic and compensation algorithmfor MEMS gyroscope, J. Transducer Technol. 23, 24-26 (2004)

13. B. Luo, W. Yin, M. Jiang, M. Wu, Temperature grads modelling of zero bias for micro mechanical gyroscope, Electron. Opt. Control 15, 29-31 (2008) 